\title{
The Doped YBCO Superconductor Crystal Structure Simulation
}

\author{
V. KAVEČANSKÝ* AND P. DIKO \\ Institute of Experimental Physics, Slovak Academy of Science, Watsonova 47, 04001 Košice, Slovak Republic \\ Pinning process plays an important role in improving utility properties of $\mathrm{YBa}_{2} \mathrm{Cu}_{3} \mathrm{O}_{7-\delta}$ (YBCO) super- \\ conductors. As a consequence of the low coherent length, the most effective pinning centers in such type of \\ materials should have nanometer size dimensions. An efficient way of introducing such centers can be a chemical \\ substitution in the YBCO lattice. Computer simulation of locally distorted YBCO crystal structure due to the \\ substitutions has been performed.
}

PACS numbers: $61.05 . \mathrm{C}-$, 74.62.Dh, 74.72.-h

\section{Introduction}

Three key factors play important roles in the superconducting state description: critical magnetic field $H_{\mathrm{c}}$, critical temperature $T_{\mathrm{c}}$ and critical current density $J_{\mathrm{c}}$. $H_{\mathrm{c}}$ and $J_{\mathrm{c}}$ depend upon two parameters that influence energy minimization, on coherence length and penetration depth. If the ratio of penetration depth to coherence length is greater than 0.7 , magnetic flux penetrates the superconductor in the form of vortices. The moving of the vortices in response to magnetic field when superconductors are to carry large currents should be restricted as otherwise it would result in appearance of resistivity. Vortices can be effectively pinned at sites of atomic defects [1]. Substituting some atoms in the YBCO lattice creates lattice distortion regions in their neighbourhoods, which become responsible for chemical pinning [2]. Crystal structure characterized by high density of lattice defects considerably deviates from a perfect periodic arrangement considered by the conventional crystallography. While the crystal defect density is not too large, the effect can be incorporated into the conventional structural model by e.g. enlarging the Debye-Waller factors and/or changing the lattice site partial occupation factors [3]. However, the process can simulate only an average distribution of site defects.

To describe the crystal structure characterized by significant periodicity deviations caused by high density of lattice distortions the local crystal structure description by means the DISCUS software $[4,5]$ has been performed. The software uses Monte Carlo statistical simulation method for minimizing the energy of a model in order to generate a disordered structure.

\section{Crystal structure simulation}

For a more detailed investigation and the crystal structure simulations the system of $\mathrm{YBa}_{2}\left(\mathrm{Cu}_{0.9} \mathrm{Ag}_{0.1}\right)_{3} \mathrm{O}_{7-\delta}$ was selected. As it was assumed that $\mathrm{Ag}$ substitutes

\footnotetext{
* corresponding author; e-mail: viktor.kavecansky@saske.sk
}

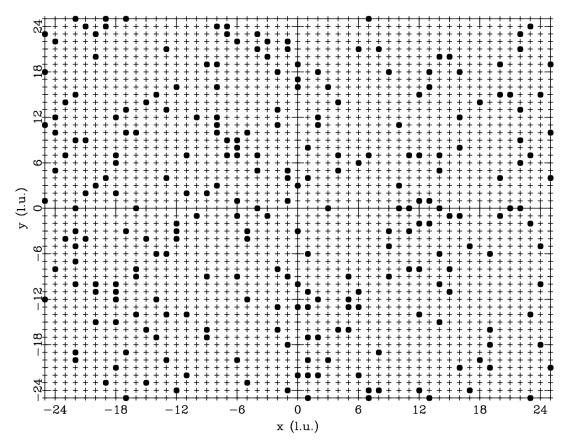

Fig. 1. Random distribution of $\mathrm{Ag}$ atoms $(\bullet)$. Basal plane cut of the $\mathrm{YBa}_{2} \mathrm{Cu}_{3} \mathrm{O}_{7-\delta}$ crystal structure (l.u. stands for lattice units).

copper atoms preferably in the $\mathrm{Cu}(1)$ positions, the simulations have been performed in the (001) basal plane of the YBCO crystal lattice.

Initially a random undistorted crystal structure was created. The (001) cut of the simulated structure is shown in Fig. 1 (for better transparency oxygen atoms $\mathrm{O}(1)$ are not shown). The $\mathrm{Ag}$ atoms distribution is completely random and is similar to the structure that can be generated by standard conventional crystallography methods.

Consecutive redistribution of $\mathrm{Ag}$ atoms was performed by creating a chemical short-range order.

The cluster creation was controlled by occupational correlation between neighbouring $\mathrm{Ag}-\mathrm{Cu}$ atoms. The chemical correlation coefficient $c_{i j}$ between a pair of sites $i$ and $j$ can be described by a relation $\left(P_{i j}-\theta^{2}\right) /$ $\theta(1-\theta)[5] . P_{i j}$ is the probability that both sites $i$ and $j$ are occupied by the same atom type and $\theta$ is its overall occupancy. The resulting structure consists of small regions characterized by an enlarged $\mathrm{Ag}$ concentration (Fig. 2).

For the simulated crystal structure shown in the figure the correlation parameters $c_{\mathrm{Cu}, \mathrm{Ag}}$ in [100], [010], and [001] directions were $0.5,0.5$, and 0.1 , respectively.

The size-effect type distortions for the $\mathrm{Cu}-\mathrm{Ag}$ pairs of atoms were simulated by the Lennard--Jones potential [5]. 


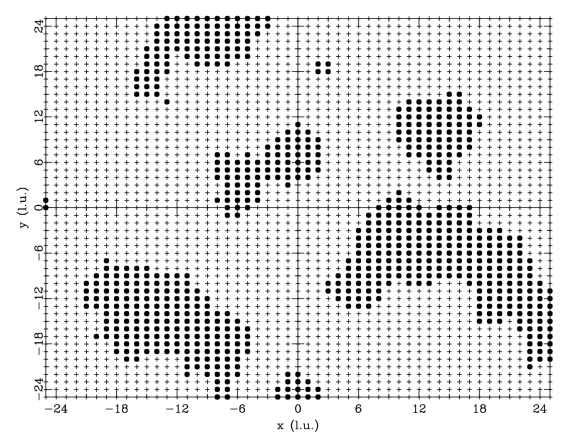

Fig. 2. Redistribution of Ag atoms by creating short-range ordered clusters.

As a result the short-range ordered crystal structure with locally distorted neighbourhoods $\mathrm{Cu}-\mathrm{Ag}$ was generated (Fig. 3).

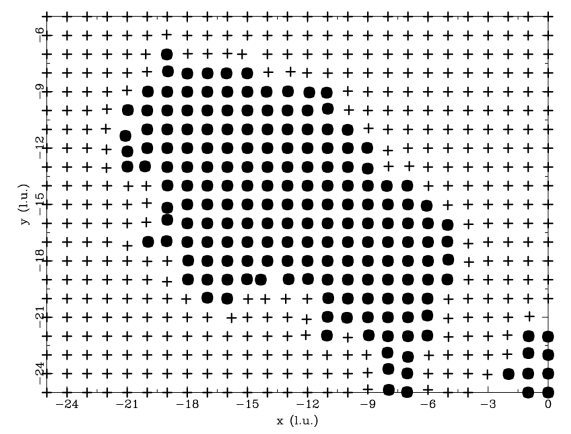

Fig. 3. Blow-up of Fig. 2 (the left bottom sector): the local partially distorted crystal structure.

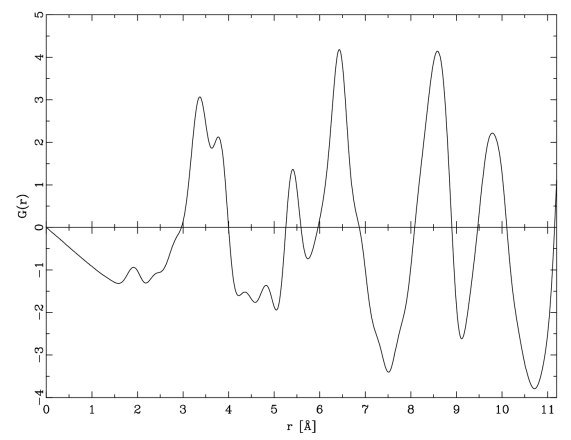

Fig. 4. Pair distribution function calculated for the corresponding simulated structure.

The scattered intensity distribution on the crystal structure created by the described simulation process (powder diffraction patterns, pair distribution function - PDF) can be consequently calculated and compared with the experimentally measured one. An example of the calculated PDF for the simulated structural model is given in Fig. 4.

\section{Summary}

The conventional crystallography is not able to describe complex materials at the length-scale of nanometer as periodicity is one of the most important assumptions of the theory. Such materials are characterized by a small randomness and local deviations from perfect periodicity. A description of these deviations can often be a fundamental part of the structural investigations. If the deviations in the atomic positions from perfect periodicity are not too large, they may be described as lattice defects. For structures characterized by a high density and overlapping of the defects such description becomes no longer useful. Such situation can be better described by means of instruments of the diffuse scattering and defect structure simulations software package as e.g. Discus. An example of such simulation for $\mathrm{YBa}_{2}\left(\mathrm{Cu}_{0.9} \mathrm{Ag}_{0.1}\right)_{3} \mathrm{O}_{7-\delta}$ was shown. The method can better describe the crystal structure with its local details and thus can significantly contribute to a better understanding of the processes taking part in such materials.

\section{Acknowledgments}

This work was realized within the frame of the projects Centre of Excellence of Advanced Materials with Nano- and Submicron Structure (ITMS 26220120019), New Materials and Technologies for Energetics (ITMS26220220061), Research and Development of Second Generation YBCO Bulk Superconductors (ITMS26220220041), which are supported by the Operational Program "Research and Development" financed through European Regional Development Fund by VEGA project No. 2/0211/10, Project ERANET-ESO and by the Centre of Excellence of Slovak Academy of Sciences NANOSMART.

\section{References}

[1] T. Nakashima, Y. Ishii, T. Katayama, H. Ogino, S. Horii, J. Shimoyama, K. Kishio J. Phys. Conf. Series $\mathbf{9 7}, 012007$ (2008).

[2] P. Diko, V. Antal, M. Kaňuchová, M. Šefčiková, J. Kováč, Supercond. Sci. Technol. 22, 1 (2009).

[3] T. Egami, S.J.L. Billinge, Underneath the Bragg Peaks, Structural Analysis of Complex Materials, Pergamon (impr. Elsevier), Amsterdam 2003.

[4] Th. Proffen, R.B. Neder, J. Appl. Crystallogr. 30, 171 (1997).

[5] R.B. Neder, Th. Proffen, Diffuse Scattering and Defect Structure Simulation, International Union of Crystallography, Oxford Science Publications, Oxford 2008. 